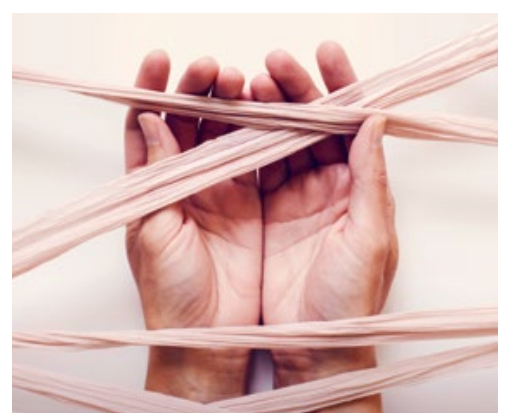

\title{
A comparison of policies and guidelines related to multimorbidity in the UK, Australia and Sri Lanka
}

\section{CPD 1 \\ Nadeeka K Chandraratne, \\ KGRV Pathirathna, Christopher Harrison, A Niroshan Siriwardena \\ Background and objectives \\ Increased rates of multimorbidity, evident in developed and developing countries, should be addressed by health policy. The aim of this study was to compare policies and guidelines related to multimorbidity in primary healthcare in countries with different health systems, to identify initiatives, gaps and opportunities for further improvement.}

\section{Methods}

We conducted a content analysis of UK, Australian and Sri Lankan policy documents and guidelines published between 2006 and 2017, in electronic databases, references and government repositories, tabulating data extracted for content, implementation plans, gaps and opportunities for development.

\section{Results}

Overall, 38 of the 56 identified documents explicitly or implicitly addressed multimorbidity or its prevention. The UK had four policy documents and guidelines specifically on multimorbidity. Australia and Sri Lanka lacked specific policies on multimorbidity, but policies did address chronic conditions and noncommunicable diseases.

\section{Discussion}

Important differences exist in how national policies seek to address multimorbidity. Policy implementation, how this affects quality of care and outcomes, and the role of primary care should be examined.

\section{LIFE EXPECTANCY has improved} dramatically over recent decades, not only in high-income countries such as Australia and the UK, but also in lowerincome and middle-income countries such as Sri Lanka; Australian and British citizens have an average life expectancy of 82.8 and 81.4 years respectively, whereas in Sri Lanka, this is 74.9 years. ${ }^{1}$ Increases in life expectancy, together with healthcare and societal changes ${ }^{2}$ have led to a greater number of people with or at risk of long-term conditions such as diabetes, mental health conditions, human immunodeficiency virus/acquired immune deficiency syndrome (HIV/AIDS) and cancer.

People living with a long-term condition often have other chronic conditions. Multimorbidity, often defined as the occurrence of two or more chronic diseases within the same person, is now the norm in older people, but is also common in younger adults. ${ }^{3}$

Multimorbidity has been rising in prevalence over recent years, ${ }^{4}$ and the rapid increase in the number of people living with chronic illness and multimorbidity, in high-income, middleincome or low-income countries, has resulted in pressure on healthcare systems worldwide. ${ }^{5}$ Multimorbidity is associated with:

- functional impairment ${ }^{6}$

- reduced quality of life ${ }^{7}$

- increased mortality

- polypharmacy resulting in higher rates of adverse drug events

- greater workload $^{8}$

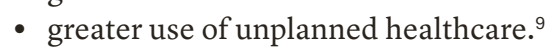

People with multimorbidity frequently receive care from primary care physicians and multiple specialists. These healthcare professionals may not communicate effectively with each other, ${ }^{10}$ leading to disorganised and fragmented care, ${ }^{11}$ and adding to patient-experienced illness burden. ${ }^{12}$

A systematic review attempting to determine the epidemiology of multimorbidity in primary care estimated the prevalence to range from $12.9 \%$ in those aged 18 years and older to $95.1 \%$ in those aged 65 years and older. ${ }^{5}$ The overall prevalence of multimorbidity in the Australian population is reported to be $25.7 \% .{ }^{13}$ In a retrospective cohort study involving people aged 18 years or older who attended general practice in England, $16 \%$ of patients had more than one chronic condition. ${ }^{14}$ A large study in Scotland found that more than $40 \%$ of the population at any age had at least one long-term condition, and almost $25 \%$ of the entire population had more than one long-term condition. ${ }^{3}$ Despite efforts to standardise terminology, ${ }^{15}$ comparisons are hampered by differences in the definition of multimorbidity. ${ }^{16}$ Estimates of prevalence in south Asia range from $4.5 \%$ to $83 \%,{ }^{17}$ but no data were available from Sri Lanka. Non-communicable diseases are estimated to account for $75 \%$ of total deaths in Sri Lanka; this estimate is lower than that for Australia (91\%) and the UK (88\%). ${ }^{18}$

Despite the prevalence of multimorbidity increasing with age, the absolute number of people with multimorbidity is higher in people aged under 65 years because of the age distribution of the population, 
particularly in areas with socioeconomic deprivation contributing to health inequalities. ${ }^{3}$ Barnett and colleagues reported that multimorbidity developed 10-15 years earlier in people living in the most deprived areas, compared with those in wealthy areas. ${ }^{3}$ In areas of socioeconomic deprivation, multiborbidity often included mental health disorders. ${ }^{3}$

The World Health Organization (WHO $)^{19}$ has highlighted the importance of a systems approach to multimorbidity and the challenges it poses for safe primary care. One of the recommendations is that awareness should be raised among policymakers and healthcare providers that multimorbidity is the norm and not the exception among people with long-term conditions. Furthermore, the WHO concluded that policies that tackle the social determinants of multimorbidity were required. ${ }^{19}$

The UK and Australia are high-income countries, well known for their strong primary healthcare systems, whereas Sri Lanka, a low-middle income country, is noted for its good health indicators. Despite economic, political and social problems, and 30 years of a bitter civil war, which ended in 2009, Sri Lanka has consistently maintained overall exemplary health indicators. In Sri Lanka, there is a life expectancy at birth of 75 years, a maternal mortality ratio of 30 per 100 , ooo live births, and an infant mortality rate of 8.5 per 1000 live births, ${ }^{20}$ which are thought to be achieved through a strong primary health system. In the UK and Sri Lanka, primary healthcare is available at no cost to patients at the point of delivery and is funded through the national healthcare system. In Australia, all citizens are covered by a universal health insurance scheme. Under Australia's scheme, the majority of general practice services (around 87\%) are provided free of charge to the patient at the point of delivery.

In the UK, general practitioners (GPs) are the key primary healthcare providers, delivering curative and preventive services for their registered populations, and functioning as gatekeepers to secondary care. GPs in Australia also provide the bulk of healthcare and act as gatekeepers to government-subsidised secondary care, but patients can see any GP they choose. In Sri Lanka, GPs do not have a gatekeeper function, with patients free to access any GP or specialist of their choice. Furthermore, doctors functioning as GPs outside the state system and in privately owned hospitals also provide primary healthcare, adding considerably to private health expenditure in Sri Lanka. Another distinct feature of the Sri Lankan primary healthcare system is the role of the medical officer of health (MOH), who is expected to deliver preventive healthcare services to a designated population.

Despite considerable differences in healthcare structure and financing, all three countries face the challenge of multimorbidity. An understanding of how each country is addressing this challenge is needed. An analysis of the national health policies that govern the practices in each country will provide an insight on the country's steps towards addressing multimorbidity and how they would be developed further to improve outcomes.

The WHO in 2016 highlighted the importance of policies referring to multimorbidity for safer health systems. ${ }^{19} \mathrm{~A}$ recent European policy analysis proposed that care for people with multimorbidity could be considerably improved with more integration and patient-centred care. This could be achieved by aligning policy, regulatory and financial environments supporting integrated care for people with multimorbidity, and through the development of multidisciplinary guidelines for multimorbidity. ${ }^{21} \mathrm{~A}$ summary report of the roundtable meeting held in October 2015 hosted by the Academy of Medical Sciences, titled 'Multiple morbidity as a global health challenge', concluded that given the universal nature of the threat, it was advisable not to consider countries separately but to share common lessons across all settings. ${ }^{22}$ The current study comparing policies relating to multimorbidity in UK, Australia and Sri Lanka is a first step to sharing experiences and learning from each other. These three countries were selected for this study because they provided examples, familiar to the authors, that emphasise primary care despite their differences in healthcare financing, systems and national income.

The aim of this study was to compare policies related to multimorbidity in primary healthcare in the UK, Australia and Sri Lanka in order to identify policy initiatives, gaps and opportunities for further improvement.

\section{Methods}

We conducted a content analysis of policy documents and guidelines issued by government and other key policymakers from the UK, Australia and Sri Lanka during the 12 years spanning 2006-17. This period was selected to ensure that relevant current policies were included in the study. The research team were academic clinicians from these three countries: two GPs, one community health specialist and a specialist in health service management.

Key search terms agreed included 'multimorbidity', 'multi-morbidity', 'complex multimorbidity', 'comorbidity', 'syndromes', 'chronic disease', 'chronic conditions', 'long-term conditions', 'non-communicable diseases', 'policy', 'programs' and 'health plans'. Two electronic databases, PubMed and Google Scholar, were searched using these key terms. Reference lists of key articles accessed were also searched. As most of the policy documents were published on government websites, as a final step, key documents and policy repositories available in the relevant countries known to the investigators were also accessed.

The policy documents and guidelines were examined for their relevance to multimorbidity in primary care, including health promotion, preventive, curative and rehabilitative interventions. Policies covering the following areas, whether explicitly or implicitly, were considered:

- specific guidelines or policy on multimorbidity

- policies or guidelines that consider multiple chronic conditions

- policies or guidelines for specific chronic conditions (eg diabetes, mental illness, cancer, HIV/AIDS, drug dependence)

- policies on medicines or drugs 
- policies or guidelines on risk factors for chronic conditions (eg alcohol, tobacco, obesity, nutrition)

- public health or health promotion

- healthcare delivery and structure

- healthcare quality and safety

- health information.

Where a specific guideline or policy was available for multimorbidity, policies that considered multiple chronic conditions in the same country were excluded to prevent duplication.

The initial selection of policies was carried out by NC and RP, and further reviewed by NS and $\mathrm{CH}$. During the validation of the policy relevance to multimorbidity, the acceptability of each document as a policy document in the national context was assessed. We then examined how these policies were translated into practice. Finally, we examined policy gaps for each country in relation to each other and the wider literature, to identify implications for further development. The data were initially extracted to a data extraction format comprising policy name, how policy was put into practice, policy gaps and opportunities for development.

\section{Results}

The search retrieved 16 documents from the UK, 22 from Australia and 18 from Sri Lanka. Of these, 13 documents from UK, 12 from Australia and 16 from Sri Lanka were scrutinised by the research team because of their relevance to multimorbidity. These documents ranged from two to more than 100 pages, providing national level policies relating directly or indirectly to care for multimorbidity, or its prevention, from the three countries under study. The number of documents that fulfilled the criteria of inclusion for each country is presented in Table 1. A detailed description of the findings of the policy documents and guidelines can be accessed in the supplementary material (available online only).

The National Institute for Health and Care Excellence (NICE) Multimorbidity: Clinical assessment and management ${ }^{23}$ was the only specific guideline on multimorbidity; Australia and Sri Lanka lacked specific documents. However, the National Strategic Framework for Chronic Conditions in Australia explicitly states that one of its objectives is to better cater for shared health determinants, risk factors and multimorbidities across a broad range of chronic conditions. This implies that the concept of multimorbidity has been integrated in the national health agenda. Sri Lanka's National Policy and Strategic Framework for Prevention and Control of Non-Communicable Disease addresses cardiovascular disease (including coronary heart disease, cerebrovascular disease and hypertension), diabetes mellitus, chronic respiratory diseases and chronic renal disease, implying the importance of addressing multiple conditions in a single policy.

All three countries had policies or guidelines for conditions such as HIV, cancer and mental health. However, conditions such as learning disabilities, chronic pain syndromes, frailty and sensory impairment were not addressed in most of the policies.

The UK has specific guidelines on multimorbidity in relation to medicines, healthcare safety and health information
(Multimorbidity: Quality standard [QS153], June 2017; ${ }^{24}$ RCGP Online services: Multimorbidity guidance for general practice March 2016; ${ }^{25}$ Multimorbidity and polypharmacy: Key therapeutic topic [KTT18], January $2017^{26}$ [not formal NICE guidance]), while the other two countries had addressed multimorbidity in their general guidelines on these aspects to varying degrees.

A promising feature observed in the Australian health system is the significance given to providing better care for marginalised and deprived citizens, initiatives that are expected to reduce health inequalities. The link between multimorbidity and deprivation or poverty, ${ }^{3}$ although well known, is not well acknowledged in policy, and few interventions are designed to address both in the UK policies.

A promising feature of most Sri Lankan policies is the high priority given to preventive and community-based approaches, which recognises that conditions are often clustered ${ }^{27}$ and that a primary care focus to multimorbidity is essential. ${ }^{28}$ Although Public Health England has recognised the importance

\section{Table 1. Summary of documents included for each country}

\begin{tabular}{|c|c|c|c|}
\hline \multirow[t]{2}{*}{ Criteria } & \multicolumn{3}{|c|}{ Number of documents retrieved } \\
\hline & UK & Australia & Sri Lanka \\
\hline Specific guidelines or policy on multimorbidity & 1 & 0 & 0 \\
\hline $\begin{array}{l}\text { Policies or guidelines that consider multiple chronic } \\
\text { conditions }\end{array}$ & NA & 1 & 1 \\
\hline $\begin{array}{l}\text { Policies or guidelines for specific chronic conditions } \\
\text { (eg diabetes, mental illness, cancer, HIV/AIDS, drug } \\
\text { dependence) }\end{array}$ & 5 & 6 & 3 \\
\hline Policies on medicines or drugs & 1 & 1 & 1 \\
\hline $\begin{array}{l}\text { Policies or guidelines on risk factors for chronic } \\
\text { conditions (eg alcohol, tobacco, obesity, nutrition) }\end{array}$ & 1 & 1 & 3 \\
\hline Public health or health promotion & 4 & 1 & 1 \\
\hline Healthcare delivery and structure & 2 & 3 & 4 \\
\hline Healthcare quality and safety & 1 & 2 & 1 \\
\hline Health information & 1 & 1 & 2 \\
\hline
\end{tabular}

HIV/AIDS, human immunodeficiency virus/acquired immune deficiency syndrome; NA, not applicable 
of determinants of multimorbidity, for example, through 'social determinants of health' (Healthy lives, healthy people: Strategy for public health in England ${ }^{29}$ ) the responsibility for much preventive work is passed to local authorities and service providers, with less emphasis on empowerment of individuals, families and communities. In Australia, there was no single policy addressing specific risk factors for multimorbidity. While the existence of health promotion policies for socially disadvantaged groups (eg Aboriginal and Torres Strait Islander peoples) is welcomed, a national public health policy on multimorbidity is lacking.

Analysis of how the policies are translated into practice revealed that, in the UK, current guidelines do not address the need for GPs to integrate their activities with third-party sectors or non-governmental organisations (NGOs), local communities or families. Although self-management is an important feature of UK health policy, there is less focus on self-management for multimorbidity. In Sri Lanka, most policies focus on infrastructure development and very low priority is given to managing patients with non-communicable diseases. The absence of an identified care provider accountable for managing patients with multimorbidity might be considered a gap in healthcare policy and systems in all three countries. However, as most primary care providers are generalists or expert generalists, it enables them to take a tailored, patientcentred care approach for people with multiple conditions.

\section{Discussion}

There are important lessons to be learned from the three countries included in this study. The UK leads the way in producing clinical guidelines that directly address the problem of multimorbidity. Australia has developed several policies that are directly concerned with the health of socially disadvantaged groups. Barnett and colleagues highlight the association of multimorbidity with socioeconomic deprivation, and the need for personalised, comprehensive continuity of care in socioeconomically deprived areas. ${ }^{3}$
Policies in Sri Lanka are more concerned with preventing chronic conditions, and on community mobilisation and empowerment. The association between lifestyle factors and development of chronic diseases is well established. Health promotion is considered the single most cost-effective intervention in addressing such risk factors, particularly for cardiovascular diseases, diabetes and cancer. ${ }^{30}$ A strong preventive healthcare structure that is supported by policies giving priority to prevention could explain Sri Lanka's good health indicator performance, despite its low economic status. However, with its demographic shift and rapidly ageing population, Sri Lanka requires specific policies or guidelines addressing multimorbidity.

These policy findings need to be considered within the context of each national health system. For example, the analysis of policies in Sri Lanka implies there is no integrated care for patients with multimorbidity, which appears to be a major vacuum. However, most medical practitioners in Sri Lanka are generalists or expert generalists, and referral to tertiary or super-specialists is minimal. Additionally, the private sector general practice system operating in Sri Lanka is considered to deliver patient-centred continuity of care in most settings. ${ }^{31}$

Identifying how practice varies from policy demands a careful study of healthcare structures and delivery in each country, which was beyond the scope of this study, and could be considered a limitation. We focused on policies implemented or active during the period 2006-17 as these were deemed relevant to the present day. However, other policies in the three countries implemented prior to 2006 may have contributed to current care for people with multimorbidity.

There are gaps in our knowledge of what types of systems or care programs are effective for people with multimorbidity. ${ }^{32}$ Most primary care trials seeking to improve outcomes for patients with multimorbidity, using complex interventions to organise care delivery differently through case management, enhanced multidisciplinary team work or greater patient and functional orientation, have shown mixed results overall, with some improvements in prescribing. ${ }^{33}$ More research is needed to develop and evaluate interventions for patients with multimorbidity, including in low-income and middle-income countries.

This study shows the importance of sharing and learning from policy differences, but further work is needed to examine how policies in different countries are translated into practice, and how this affects the quality of health service provision and outcomes. Whether a move towards greater generalism in primary and secondary healthcare provision will help meet the needs of patients with multimorbidity should also be explored. Perhaps key lessons for these countries are that Sri Lanka needs to focus on policies that emphasise integration and patient-centred healthcare delivery for people with multimorbidity. In the UK and Australia, policies on community-based approaches to address determinants of multimorbidity are needed.

\section{Authors}

Nadeeka K Chandraratne MBBS, MSc, MDS, $\mathrm{MD}$, International Fellow, Community and Health Research Unit, University of Lincoln, UK; Senior Registrar in Community Medicine, Ministry of Health Sri Lanka.nChandraratne@lincoln.ac.uk

KGRV Pathirathna MBBS, MSc, MD, International Fellow, Community and Health Research Unit, University of Lincoln, UK; Senior Registrar in Medical Administration, Ministry of Health, Sri Lanka Christopher Harrison B Psych (Hons), MSocHlth, PhD, Research Fellow, Menzies Centre for Health Policy, Sydney School of Public Health, Australia A Niroshan Siriwardena MBBS, MMedSci, PhD, FRCGP, Professor of Primary and Prehospital Health Care, Community and Health Research Unit, University of Lincoln, UK

Competing interests: Professor Siriwardena has received a grant from Galen Pharmaceuticals, outside the submitted work.

Provenance and peer review: Commissioned, externally peer reviewed.

\section{References}

1. World Health Organization. Life expectancy at birth (years), 2000-2015. Geneva: WHO, 2015.

2. van Oostrom SH, Gijsen R, Stirbu I, et al. Time trends in prevalence of chronic diseases and multimorbidity not only due to aging: Data from general practices and health surveys. PLoS One 2016;11(8):e0160264.

3. Barnett K, Mercer SW, Norbury M, Watt G, Wyke S, Guthrie B. Epidemiology of multimorbidity and implications for health care, research, and medical education: A cross-sectional study. Lancet 2012;380(9836):37-43. 
4. Uijen AA, van de Lisdonk EH. Multimorbidity in primary care: Prevalence and trend over the last 20 years. Eur J Gen Pract 2008;14 Suppl 1:28-32.

5. Violan C, Foguet-Boreu Q, Flores-Mateo G, et al. Prevalence, determinants and patterns of multimorbidity in primary care: A systematic review of observational studies. PLoS One 2014;9(7):e102149.

6. Kadam UT, Croft PR, North Staffordshire GP Consortium Group. Clinical multimorbidity and physical function in older adults: A record and health status linkage study in general practice. Fam Pract 2007;24(5):412-19.

7. Fortin M, Lapointe L, Hudon C, Vanasse A, Ntetu AL, Maltais D. Multimorbidity and quality of life in primary care: A systematic review. Health Qual Life Outcomes 2004;2:51.

8. Buffel du Vaure C, Ravaud P, Baron G, Barnes C, Gilberg S, Boutron I. Potential workload in applying clinical practice guidelines for patients with chronic conditions and multimorbidity: A systematic analysis. BMJ Open 2016;6(3):e010119.

9. Harrison C, Britt H, Miller G, Henderson J. Multimorbidity. Aust Fam Physician 2013;42(12):845

10. Farmer C, Fenu E, O'Flynn N, Guthrie B. Clinical assessment and management of multimorbidity: Summary of NICE guidance. BMJ 2016;354:i4843.

11. Sinnott C, Mc Hugh S, Browne J, Bradley C. GPs' perspectives on the management of patients with multimorbidity: Systematic review and synthesis of qualitative research. BMJ Open 2013;3(9):e003610.

12. Rosbach M, Andersen JS. Patient-experienced burden of treatment in patients with multimorbidity - A systematic review of qualitative data. PLoS One 2017;12(6):e0179916.

13. Harrison C, Henderson J, Miller G, Britt H. The prevalence of diagnosed chronic conditions and multimorbidity in Australia: A method for estimating population prevalence from general practice patient encounter data. PLoS One 2017;12(3):e0172935.

14. Salisbury C, Johnson L, Purdy S, Valderas JM Montgomery AA. Epidemiology and impact of multimorbidity in primary care: A retrospective cohort study. Br J Gen Pract 2011;61(582):e12-21.

15. Le Reste JY, Nabbe P, Rivet C, et al. The European general practice research network presents the translations of its comprehensive definition of multimorbidity in family medicine in ten European languages. PLoS One 2015;10(1):e0115796.

16. Willadsen TG, Bebe A, Køster-Rasmussen R, et al. The role of diseases, risk factors and symptoms in the definition of multimorbidity A systematic review. Scand J Prim Health Care 2016;34(2):112-21.

17. Pati $S$, Swain $S$, Hussain MA, et al. Prevalence and outcomes of multimorbidity in South Asia: A systematic review. BMJ Open 2015;5(10):e007235.

18. World Health Organization. Noncommunicable diseases country profiles. Geneva: WHO, 2014.

19. World Health Organization. Multimorbidity: Technical series on safer primary care. Geneva: WHO, 2016.

20. World Health Organization. Global Health Observatory (GHO) data. Geneva: WHO, 2016. Available at www.who.int/gho/countries/lka/ country_profiles/en [Accessed 18 December 2017].

21. Rijken M, Struckmann V, Van der Heide I, et al. How to improve care for people with multimorbidity in Europe? Geneva: World Health Organization, 2017.
22. Sciences TAoM. Multiple morbidities as a global health challenge: Summary of a roundtable meeting held on 7 October 2015 hosted by the Academy of Medical Sciences. London: Sciences TAoM, 2015.

23. National Institute for Health and Care Excellence. Multimorbidity: Clinical assessment and management. London: NICE, 2016. Available at www.nice.org.uk/guidance/NG56 [Accessed 18 December 2017].

24. National Institute for Health and Care Excellence. Multimorbidity: Quality standard [QS153]. London: NICE, 2017. Available at www.nice.org.uk/ guidance/qs153 [Accessed 18 December 2017].

25. Royal College of General Practitioners. Online services: Multimorbidity guidance for general practice. London: RCGP, 2017. Available at http://elearning.rcgp.org.uk/mod/page/view. php?id=4775 [Accessed18 December 2017].

26. National Institute for Health and Care Excellence. Multimorbidity and polypharmacy: Key therapeutic topic [KTT18]. London: NICE, 2017. Available at www.nice.org.uk/advice/ktt18 [Accessed 18 December 2017]

27. Mino-León D, Reyes-Morales H, Doubova SV, Pérez-Cuevas R, Giraldo-Rodríguez L, AgudeloBotero M. Multimorbidity patterns in older adults: An approach to the complex interrelationships among chronic diseases. Arch Med Res 2017;48(1):121-27.

28. Starfield B. Global health, equity, and primary care. J Am Board Fam Med 2007;20(6):511-13.

29. Department of Health, UK. Healthy lives, healthy people: Strategy for public health in England. London: Crown copyright, 2010. Available at www. gov.uk/government/uploads/system/uploads/ attachment_data/file/216096/dh_127424.pdf [Accessed 18 December 2017].

30. Puska P, Vartiainen E, Nissinen A, Laatikainen $T$, Jousilahti P. Background, principles, implementation, and general experiences of the North Karelia Project. Glob Heart 2016;11(2):173-78.

31. Govindaraj R, Navaratne K, Cavagnero E Seshadri SR. Health care in Sri Lanka: What can the private health sector offer? Health, nutrition, and population (HNP) discussion paper. New Delhi: HNP, 2014.

32. Hopman P, de Bruin SR, Forjaz MJ, et al. Effectiveness of comprehensive care programs for patients with multiple chronic conditions or frailty: A systematic literature review. Health Policy 2016;120(7):818-32.

33. Smith SM, Wallace E, O'Dowd T, Fortin M Interventions for improving outcomes in patients with multimorbidity in primary care and community settings. Cochrane Database Syst Rev 2016;3:CD006560. 